\title{
The generation of connected high and very high energy $\gamma$-rays and radio emission in active galactic nuclei
}

\begin{abstract}
Z. Osmanov
Centre for Theoretical Astrophysics, ITP, Ilia State University, 0162 Tbilisi, Georgia

e-mail: z.osmanov@astro-ge.org

Received 2 April 2010 / Accepted 21 March 2011

\section{ABSTRACT}

Aims. We consider relativistic electrons in magnetospheric flows close to a supermassive black hole and study the mechanism of quasi-linear diffusion (QLD) to investigate the correlation between $\gamma$-ray and radio emission in active galactic nuclei.

Methods. Moving in the nonuniform magnetic field, the particles experience a force that is responsible for the conservation of the adiabatic invariant. This force, together with the radiation reaction force, tends to decrease the pitch angles. Contrary to this, the QLD attempts to increase the pitch angle, and this maintains the synchrotron emission regime. To examine the balance between the QLD and the aforementioned dissipative factors we investigate the quasi-stationary state by applying the kinetic equation.

Results. Considering the magnetospheric plasma close to the supermassive black hole, we examined the efficiency of the QLD for different parameters. By examining the cyclotron instability, we show that despite the efficient dissipative factors, the cyclotron modes excite transverse and longitudinal-transversal waves, which lead to the QLD. We find that the QLD provides a connection of emission in the $\gamma$-ray and radio domains. We show that under favourable conditions the radio emission from $22 \mathrm{MHz}$ to $9 \mathrm{GHz}$ on the mpc scale is associated with $\gamma$-ray emission from $900 \mathrm{GeV}$ down to $9 \mathrm{GeV}$ on the same scale.
\end{abstract}

Key words. galaxies: active - instabilities - magnetohydrodynamics (MHD) - radiation mechanisms: non-thermal

\section{Introduction}

During the last decades the interest in very high energy (VHE) radiation of active galactic nuclei (AGNs) has considerably increased because of the detailed data from the new telescopes (MAGIC, HESS, EGRET, Fermi). Active galactic nuclei, and especially blazars reveal a strong connection between high-energy $\gamma$-ray and radio emission. Since the EGRET era, a direct connection between radio and $\gamma$-ray flux density has been intensively discussed. In particular, Bloom (2008) performed a statistical analysis of the broadband properties of EGRET blazars and showed a strong positive correlation between the total radio luminosity at $8.4 \mathrm{GHz}$ and the $\gamma$-ray luminosity in the EGRET band $\left(L_{\gamma} \sim L_{\text {rad }}^{0.77}\right)$. The comparison of the radio flux density at $8.4 \mathrm{GHz}$ and the $\gamma$-ray $(>100 \mathrm{MeV})$ photon flux measured by the Fermi $\gamma$-ray Space Telescope has been presented by Giroletti et al. (2010) and the Spearman's rank correlation coefficient was estimated to be 0.57. Kovalev et al. (2009) found a positive correlation between the pc-scale radio flux at $15 \mathrm{GHz}$ and the $\gamma$-ray photon flux in a sub-band of the Fermi/LAT detector $(100 \mathrm{MeV}-1 \mathrm{GeV})$, and a time separation of a few months between $\gamma$-ray and radio flares.

Most commonly the VHE emission of AGNs is explained in terms of the inverse Compton upscattering (Blandford et al. 1990) and curvature radiation (Gangadhara 1996; Thomas \& Gangadhara 2005). The major reason for this is that if the magnetic field is strong, the synchrotron cooling timescale is very short and relativistic particles very soon transit to their ground Landau level, which prevents the subsequent emission process (Osmanov \& Machabeli 2010).

Recently, it has been shown that the quasi-linear diffusion (QLD) may create necessary conditions in the magnetospheres of AGNs and pulsars for avoiding the synchrotron emission damping despite the strong magnetic field. According to the model of Kazbegi et al. (1992), the cyclotron instability appears in the magnetospheres of pulsars. These unstable modes lead to the feedback of the excited waves on particles by means of the process of the diffusion. The pitch angles are no longer negligible and the emission process continues (e.g. Machabeli \& Usov 1979; Lominadze et al. 1979; Malov \& Machabeli 2001; Chkheidze \& Machabeli 2007; Chkheidze et al. 2011). This model was applied to the Crab pulsar (Machabeli \& Osmanov 2009, 2010; Chkheidze et al. 2011) to explain the recent results from the MAGIC Cherenkov telescope between 2007 October and 2008 February (Albert et al. 2002).

In the context of works presented by Bloom (2008) and Giroletti et al. (2010) (where the observationally evident strong correlation of high-energy $\gamma$-rays and low-frequency radiation is discussed), it is of fundamental importance that according to the QLD, two different radiation domains are excited. As was studied by Osmanov \& Machabeli (2010) and Osmanov (2010), the cyclotron resonance efficiently generates transverse momenta, which leads to non-zero pitch angles and the subsequent synchrotron radiation. This means that a connection between two radiation domains is naturally introduced into the quasi-linear diffusion process. In particular, examining the QLD in AGNs, Osmanov \& Machabeli (2010) studied the generation of X-rays associated with the radio emission, and the work of Osmanov (2010) was related to high-energy emission associated with submillimeter/infrared radiation.

In this paper we study the production of high-energy radiation in the GeV-TeV interval in the magnetospheres of supermassive black holes. The paper is organized as follows. In Sect. 2 we present the model, in Sect. 3 we apply the mechanism to AGNs and in Sect. 4 we summarize our results. 


\section{Theory}

In general, Lorentz factors of magnetospheric plasma particles in $\mathrm{AGNs}^{1}$ lie in a broad interval ranging from $\sim 1$ up to $\sim 10^{8}$ (Osmanov et al. 2007; Rieger \& Aharonian 2008), therefore, for simplicity one can consider the magnetosphere to be composed of the electron-positron plasma component with relatively low Lorentz factors, $\gamma_{\mathrm{p}}$, and highly relativistic electrons, the so-called beam component electrons with the Lorentz factor $\gamma_{\mathrm{b}}$ $\left(\gamma_{\mathrm{b}} \gg \gamma_{\mathrm{p}}\right)$.

In this section we consider the QLD mechanism and derive an average value of the pitch angles driven by the diffusion. In general the process of energy dissipation that takes place in strong magnetic fields is dynamically controlled by two forces: one is the synchrotron radiative force (Landau \& Lifshitz 1971)

$F_{\perp}=-\alpha \psi\left(1+\gamma_{\mathrm{b}}^{2} \psi^{2}\right), \quad F_{\|}=-\alpha \gamma_{\mathrm{b}}^{2} \psi^{2}$,

where $\alpha=2 e^{2} \omega_{B}^{2} /\left(3 c^{2}\right), \psi$ is the pitch angle, $\omega_{B} \equiv e B / m c$ is the cyclotron frequency, $B$ is the magnetic induction, $c$ is the speed of light, $e$ and $m$ are electron's charge and the rest mass, respectively; the other is the force $\boldsymbol{G}$, responsible for the conservation of the adiabatic invariant, $I=3 c p_{\perp}^{2} / 2 e B$ (Landau \& Lifshitz 1971)

$G_{\perp}=-\frac{m c^{2}}{\rho} \gamma_{\mathrm{b}} \psi, \quad G_{\|}=\frac{m c^{2}}{\rho} \gamma_{\mathrm{b}} \psi^{2}$,

where $\rho$ is the curvature radius of the magnetic field lines. In general, in the magnetospheres of AGNs two effects take place: the energy dissipation provoked by the forces, $\boldsymbol{F}, \boldsymbol{G}$, which lead to the decrease of the pitch angles, the QLD, which opposes the first effect. The dynamical process saturates when the effects of the above-mentioned forces are balanced by the diffusion, which leads to non-zero pitch angles and the subsequent radiation.

As discussed by Machabeli \& Usov (1979), Machabeli \& Osmanov (2010), Osmanov \& Machabeli (2010), for an activation of the QLD, the cyclotron modes must be excited, which leads to the diffusion in terms of feedback of the cyclotron waves on particles. On the other hand, according to the work of Kazbegi et al. (1992), the anomalous Doppler effect can efficiently induce unstable cyclotron waves with the corresponding frequency (Machabeli \& Usov 1979; Osmanov \& Machabeli 2010)

$v \approx \frac{\omega_{B}}{2 \pi \delta \cdot \gamma_{\mathrm{b}}}$

where $\delta=\omega_{\mathrm{p}}^{2} /\left(4 \omega_{B}^{2} \gamma_{\mathrm{p}}^{3}\right), \omega_{\mathrm{p}} \equiv \sqrt{4 \pi n_{\mathrm{p}} e^{2} / m}$ is the plasma frequency and $n_{\mathrm{p}}$ is the plasma density. Here we assume that energy in plasma is uniformly distributed, therefore, the plasma density can be approximated to be $n_{\mathrm{b}} \gamma_{\mathrm{b}} / \gamma_{\mathrm{p}}$, where $n_{\mathrm{b}}$ is the beam density (Machabeli \& Osmanov 2009). Although the aforementioned expression is a direct consequence of a resonance character of the cyclotron instability, in exciting waves all resonance particles (with broad energy spectra) participate, therefore the range of spectral frequencies is wide and the waves are not characterized by a monochromatic signature. In the framework of the present approach, one has two simultaneously generated connected VHE $\gamma$-rays and low-frequency cyclotron modes. This result seems to be promising in the context of recent investigations,

\footnotetext{
1 The magnetosphere of AGNs with their typical lengthscales $10^{14-15} \mathrm{~cm}$ is a region around the supermassive black hole, where the magnetic field $\left(10^{4} \mathrm{G}-\right.$ close to the black hole and $10-100 \mathrm{G}-$ in the light cylinder zone) is dominant.
}

because it shows the strong connection between the $\gamma$-ray and radio fluxes (Bloom 2008; Giroletti et al. 2010).

A similar problem was studied by Osmanov \& Machabeli (2010) who examined the physical regime $\left|G_{\perp}\right| \gg\left|F_{\perp}\right|$ and $\left|G_{\|}\right| \ll\left|F_{\|}\right|$and studied the production of X-rays $(0.13-100 \mathrm{keV})$ connected with the radio emission (0.04-35 MHz). A different regime, $\left|G_{\perp}\right| \ll\left|F_{\perp}\right|$ and $\left|G_{\|}\right| \ll\left|F_{\|}\right|$, was examined by Osmanov (2010). This regime shows the possibility of production of $\mathrm{MeV}-$ $\mathrm{GeV} \gamma$-rays, which are strongly correlated with submillimeter/infrared radiation domains. In the present paper we examine the same regime, but for different parameters. Unlike the previous work, where the QLD was studied in the light cylinder area where the magnetic field is of the order of $\sim 300 \mathrm{G}$, in the present paper the physics of the QLD is examined for a region close to the supermassive black hole event horizon where $B \sim 10^{4} \mathrm{G}$ (see Thorne et al. 1988). Another difference is the fact that here we study the cyclotron waves generated by plasma particles with Lorentz factors, $\gamma_{\mathrm{p}} \sim 2-4$, whereas values of $\gamma_{\mathrm{p}}$ considered by Osmanov (2010) were higher by two orders of magnitude. As we will see, the aforementioned differences significantly change the results. By comparing the transverse and longitudinal components of the forces, one obtains

$$
\begin{aligned}
& \frac{G_{\perp}}{F_{\perp}} \approx 5 \times 10^{-8} \times\left(\frac{10^{8}}{\gamma_{\mathrm{b}}}\right) \times\left(\frac{10^{4} \mathrm{G}}{B}\right)^{2} \times\left(\frac{10^{-2} \mathrm{rad}}{\psi}\right)^{2} \times\left(\frac{R_{\mathrm{g}}}{\rho}\right), \\
& \frac{\left|G_{\|}\right|}{\left|F_{\|}\right|} \approx 5 \times 10^{-12} \times\left(\frac{10^{8}}{\gamma_{\mathrm{b}}}\right) \times\left(\frac{10^{4} \mathrm{G}}{B}\right)^{2} \times\left(\frac{R_{\mathrm{g}}}{\rho}\right) .
\end{aligned}
$$

Evidently, the relations $\left|G_{\perp}\right| \ll\left|F_{\perp}\right|$ and $\left|G_{\|}\right| \ll\left|F_{\|}\right|$are satisfied if $\psi \gg 2.2 \times 10^{-6} \mathrm{rad}$ if we consider the physical parameters $\gamma_{\mathrm{b}} \sim 10^{8}, B \sim 10^{4} \mathrm{G}, R_{\mathrm{g}} \sim \rho$.

By assuming a quasi-stationary scenario $(\partial / \partial t=0)$, the corresponding kinetic equation will be different from that of Machabeli \& Osmanov (2010)

$$
\begin{aligned}
& \frac{1}{m c \gamma_{\mathrm{b}} \psi} \frac{\partial}{\partial \psi}\left(\psi F_{\perp} f\right)+\frac{1}{m c} \frac{\partial}{\partial \gamma_{\mathrm{b}}}\left(F_{\|} f\right)+v \frac{\partial f}{\partial r}= \\
& \frac{1}{m^{2} c^{2} \gamma_{\mathrm{b}}^{2} \psi} \frac{\partial}{\partial \psi}\left(\psi D_{\perp \perp} \frac{\partial f}{\partial \psi}\right)+\frac{1}{m c \psi} \frac{\partial}{\partial \psi}\left(\psi^{2} D_{\perp \|} \frac{\partial f}{\partial \gamma_{\mathrm{b}}}\right) \\
&+\frac{1}{m c} \frac{\partial}{\partial \gamma_{\mathrm{b}}}\left(\psi D_{\perp \|} \frac{\partial f}{\partial \psi}\right),
\end{aligned}
$$

where $f=f\left(\psi, \gamma_{\mathrm{b}}\right)$ is the distribution function of particles,

$D_{\perp \perp} \approx \frac{\pi^{2} e^{2}}{m^{2} c^{3}} \frac{\delta}{\gamma_{\mathrm{b}}^{2}}\left|E_{k}\right|^{2}, \quad D_{\perp_{\|}} \approx-\frac{\pi e^{2}}{4 m c^{2} \gamma_{\mathrm{b}}}\left|E_{k}\right|^{2}$,

are the diffusion coefficients and $\left|E_{k}\right|^{2}$ is the energy density per unit wavelength.

For estimating $\left|E_{k}\right|^{2}$, we assume that half of the plasma energy density, $m c^{2} n_{\mathrm{b}} \gamma_{\mathrm{b}} / 2$, converts to the energy density of the waves $\left|E_{k}\right|^{2} k$, then an expression of $\left|E_{k}\right|^{2}$ writes as follows

$\left|E_{k}\right|^{2}=\frac{m c^{3} n_{\mathrm{b}} \gamma_{\mathrm{b}}}{2 \omega}$

For solving the aforementioned kinetic equation with respect to the pitch angles we separate the variables by expressing the distribution function as $\chi(\psi) f\left(\gamma_{\mathrm{b}}\right)$. Then the equation governing the distribution by pitch angles is given by

$$
\frac{\partial}{\partial \psi}\left(\psi F_{\perp} f\right)=\frac{1}{m c \gamma_{\mathrm{b}}} \frac{\partial}{\partial \psi}\left(\psi D_{\perp \perp} \frac{\partial f}{\partial \psi}\right),
$$


and the corresponding solution writes as

$\chi(\psi)=C \mathrm{e}^{-A \psi^{4}}$,

where

$A \equiv \frac{\alpha m c \gamma_{\mathrm{b}}^{3}}{4 D_{\perp \perp}}$

For simplicity we use the average value of the pitch angle, which after taking Eqs. $(10,11)$ into account leads to

$\bar{\psi}=\frac{\int_{0}^{\infty} \psi \chi(\psi) \mathrm{d} \psi}{\int_{0}^{\infty} \chi(\psi) \mathrm{d} \psi} \approx \frac{0.5}{\sqrt[4]{A}}$

Clearly, despite very efficient synchrotron losses the quasi-linear diffusion works against the dissipative forces and maintains nonzero pitch angles, which prevent the synchrotron mechanism from damping. Under these conditions, the relativistic particles with Lorentz factors, $\gamma$, radiate in the synchrotron regime and emit photons with energies (Rybicki \& Lightman 1979)

$\epsilon_{\mathrm{eV}} \approx 1.2 \times 10^{-8} B \gamma^{2} \sin \psi$

\section{Results}

According to the observational evidence, AGNs are characterized by emission in the VHE domain, which cannot be explained in the framework of thermal processes. This can also be explained through the existence of ultra-relativistic particles in the magnetosphere. In general, there are several acceleration mechanisms that might provide very high Lorentz factors. We show that processes such as Fermi-type acceleration (Catanese $\&$ Weekes 1999) and acceleration owing the the black hole dynamo mechanism (Levinson 2000) can provide Lorentz factors of the order of $\sim 10^{7-9}$ close to the supermassive black holes, i.e. at $r \sim R_{\mathrm{g}}$, where $R_{\mathrm{g}} \equiv 2 G M_{\mathrm{BH}} / c^{2} \approx 3 M_{9} \times 10^{14} \mathrm{~cm}$ is the gravitational radius of the black hole, $M_{9} \equiv M /\left(10^{9} M_{\odot}\right)$ and $M_{\odot}$ is the solar mass.

Osmanov \& Machabeli (2010) have shown that the synchrotron cooling timescale for ultra-relativistic electrons in the magnetospheres of AGNs close to the black hole is of the order of $\sim 10^{-5}-10^{-4} \mathrm{~s}$. To estimate the efficiency of the energy losses it is reasonable to calculate the kinematic timescale $t_{\text {kin }} \sim R_{\mathrm{g}} / c$. Considering the supermassive black hole with $M_{9}=1$, one can see that $t_{\text {kin }} \sim 10^{4} \mathrm{~s}$, which by many orders of magnitude exceeds the dissipation timescale, therefore, the cooling process is extremely efficient and the electrons stop emitting very soon.

Anomalous Doppler effect significantly changes the overall radiative pattern of the system. Equation (3) clearly shows that the mentioned effect efficiently induces unstable cyclotron waves with the frequency (Osmanov \& Machabeli 2010)

$v \approx 8.7 \times 10^{7}\left(\frac{\gamma_{\mathrm{p}}}{2}\right)^{4} \times\left(\frac{10^{8}}{\gamma_{\mathrm{b}}}\right)^{2} \times\left(\frac{B}{10^{4} \mathrm{G}}\right)^{3} \times\left(\frac{20 \mathrm{~cm}^{-3}}{n_{\mathrm{b}}}\right) \mathrm{Hz}$

which in turn lead to the feedback of these modes to the particles and switch on the QLD, which generates the non-vanishing pitch angles and the subsequent synchrotron process.

As a first example, we examine a supermassive black hole with $M_{9}=1$ and the typical value of the magnetic induction for a nearby zone of the black hole $B=10^{4} \mathrm{G}$. Since we assume that energy in plasma is uniformly distributed, the value of plasma density $n_{\mathrm{b}} \gamma_{\mathrm{b}} / \gamma_{\mathrm{p}}$, for $n_{\mathrm{b}}=20 \mathrm{~cm}^{-3}$ and $B=10^{4} \mathrm{G}$ is of the order

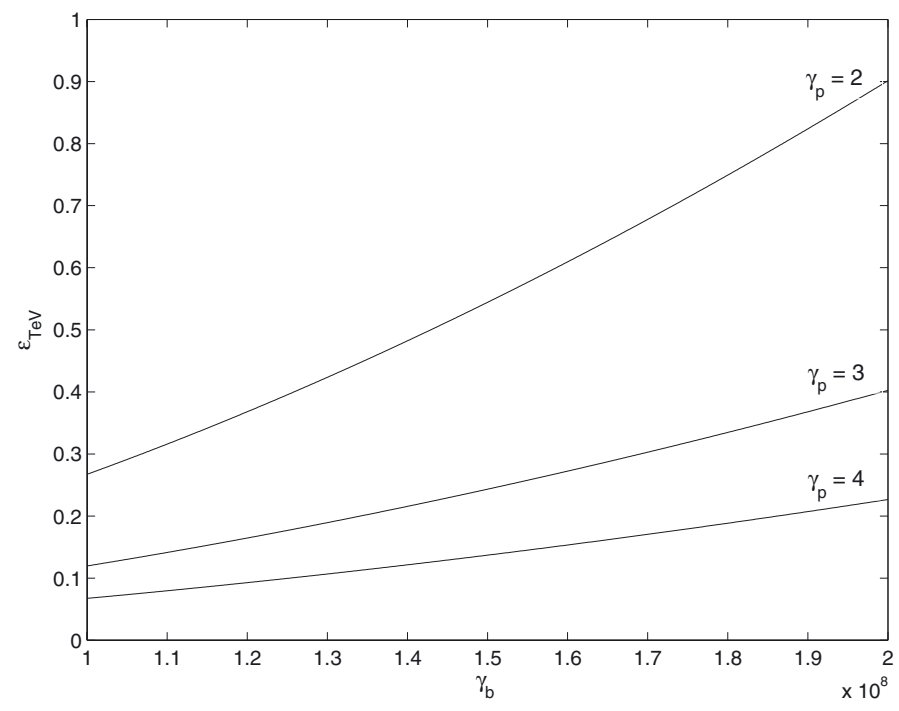

Fig. 1. Behaviour of $\epsilon_{\mathrm{TeV}}$ with respect to $\gamma_{\mathrm{b}}$. The set of parameters is $M_{9}=1, B=10^{4} \mathrm{G}, n_{\mathrm{b}}=20 \mathrm{~cm}^{-3}$ and $\gamma_{\mathrm{p}} \in\{2 ; 3 ; 4\}$.

of $10^{9} \mathrm{~cm}^{-3}$, which is compatible with observations of narrow and broad line regions and indicates that the plasma density in the environment of AGNs lies in the interval $\sim\left(10^{5}-10^{14}\right) \mathrm{cm}^{-3}$ (Xu et al. 2007; Arav et al. 2002). We consider the beam component's Lorentz factors to be of the order of $10^{8}$ and the plasma component with the Lorentz factor, $\gamma_{\mathrm{p}}=2$. From Eq. (14) one can show that the cyclotron frequency of the order of $87 \mathrm{MHz}$ is excited, which in turn, by means of the QLD, leads to the average value of the pitch angle $\sim 0.2 \mathrm{rad}$ (see Eqs. (11), (12)) and the subsequent synchrotron emission in the VHE domain $\sim 240 \mathrm{GeV}$ (see Eq. (13)).

In Fig. 1 we show the dependence of $\epsilon_{\mathrm{TeV}}$ on $\gamma_{\mathrm{b}}$. The set of parameters is $M_{9}=1, B=10^{4} \mathrm{G}, n_{\mathrm{b}}=20 \mathrm{~cm}^{-3}$ and $\gamma_{\mathrm{p}} \in\{2 ; 3 ; 4\}$. Clearly, physically realistic parameters might guarantee the synchrotron emission from $\sim 70 \mathrm{GeV}\left(\gamma_{\mathrm{b}}=10^{8}, \gamma_{\mathrm{p}}=4\right)$ to $0.9 \mathrm{TeV}$ $\left(\gamma_{\mathrm{b}}=2 \times 10^{8}, \gamma_{\mathrm{p}}=2\right)$. These plots show a continuously increasing character of $\epsilon_{\mathrm{TeV}}$ with respect to $\gamma_{\mathrm{b}}$, which is a natural result of the fact that more energetic beam particles radiate photons with higher energies. Unlike the beam Lorentz factors, the behaviour of $\epsilon_{\mathrm{TeV}}$ with respect to $\gamma_{\mathrm{p}}$ is a continuously decreasing function. This can be seen from Eqs. (11), (12): $\bar{\psi} \sim \sqrt[4]{D_{\perp \perp}}$, which by combining with Eqs. (7), (8) reduces to $\bar{\psi} \sim 1 / \gamma_{\mathrm{p}}^{2}$. Therefore, by considering higher values of $\gamma_{p}$, the resulting synchrotron emission energy will be lower.

In Fig. 2 we show the behaviour of $\epsilon_{\mathrm{TeV}}$ with respect to $v$ and $\gamma_{\mathrm{p}}$. The set of parameters is $M_{9}=1$ and $B=10^{4} \mathrm{G}$, $n_{\mathrm{b}}=20 \mathrm{~cm}^{-3}$. The 2D surface shows that for lower values of the plasma Lorentz factors, the synchrotron emission energy is higher and the corresponding cyclotron frequency relatively is lower. Figure 3 shows the dependance of $\epsilon_{\mathrm{TeV}}$ on the cyclotron radio frequency for three different values of plasma Lorentz factors $\gamma_{\mathrm{p}}=\{2 ; 3 ; 4\}$. Evidently, the VHE $\gamma$-rays in the TeV domain are connected with the radio emission at $\sim 87 \mathrm{MHz}$, and the higher frequency radio emission $\sim 1.4 \mathrm{GHz}$ is connected with the lower energy $(\sim 70 \mathrm{GeV}) \gamma$-ray synchrotron emission.

One of the major magnetospheric parameters is the density of the beam component. In Fig. 4 we show the behaviour of $\epsilon_{\mathrm{TeV}}$ versus $n_{\mathrm{b}}$. The set of parameters is $M_{9}=1$ and $B=10^{4} \mathrm{G}$, $\gamma_{\mathrm{p}}=2$ and $n_{\mathrm{b}}=\{0.2 ; 2 ; 20\} \mathrm{cm}^{-3}$. Obviously, the higher the beam density, the higher the $\gamma$-ray synchrotron radiation energy. 


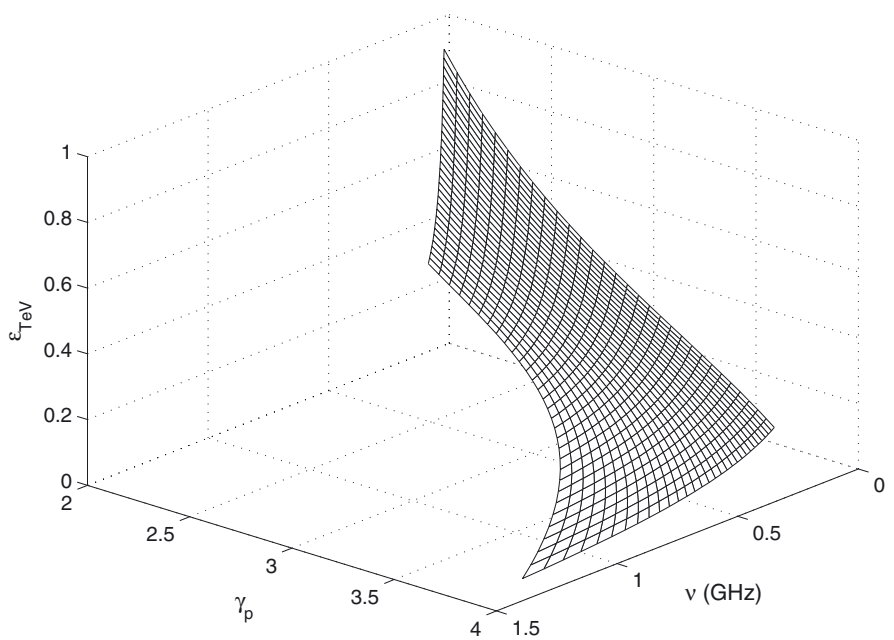

Fig. 2. Behaviour of $\epsilon_{\mathrm{TeV}}$ versus $v$ and $\gamma_{\mathrm{p}}$. The set of parameters is $M_{9}=1$ and $B=10^{4} \mathrm{G}, n_{\mathrm{b}}=20 \mathrm{~cm}^{-3}$.

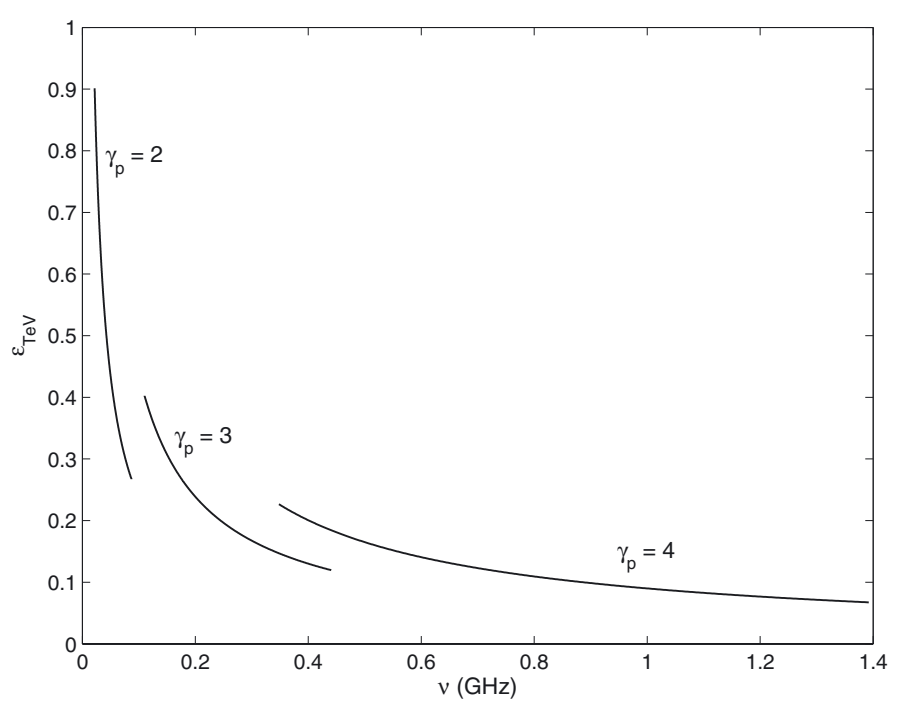

Fig. 3. Behaviour of $\epsilon_{\mathrm{TeV}}$ versus $v$. The set of parameters is $M_{9}=1$ and $B=10^{4} \mathrm{G}, n_{\mathrm{b}}=20 \mathrm{~cm}^{-3}$ and $\gamma_{\mathrm{p}}=\{2 ; 3 ; 4\}$. Evidentlythe curves are truncated, which is a direct result of the fact that the curves are onedimensional sections of a two-dimensional surface.

As we already mentioned, the average value of the pitch angle is proportional to $\sqrt[4]{D_{\perp \perp}}$, which by combining with $D_{\perp \perp} \sim n_{\mathrm{b}}^{3}$ (see Eqs. (7), (8)) confirms the results shown in the plots. For the considered interval of $n_{\mathrm{b}}$ the photon energy varies in the range, 8.5 GeV-0.9 TeV.

Figure 5 presents the dependance of $\epsilon_{\mathrm{TeV}}$ on $v$. From the plots we see that the $\mathrm{TeV}$ emission is connected to the radio band $\sim 22 \mathrm{MHz}$ and the high-energy emission, $8.5 \mathrm{GeV}$ is connected to $\sim 8.7 \mathrm{GHz}$ radio domain. The present results are different from those of Osmanov \& Machabeli (2010) and Osmanov (2010), where a different parametric space, has been considered. In particular, Osmanov \& Machabeli (2010) examined $M_{9}=1, \gamma_{\mathrm{p}}=2, \gamma_{\mathrm{b}} \sim 10^{6-9}, n_{\mathrm{b}}=2000 \mathrm{~cm}^{-3}$ and Osmanov (2010) considered $M_{9}=1, \gamma_{\mathrm{p}} \in\{200 ; 250 ; 300\}, \gamma_{\mathrm{b}} \sim 10^{8}$ and $n_{\mathrm{b}}=\{5 ; 10 ; 15\} \mathrm{cm}^{-3}$.

According to the quasi-linear approach, the efficiency of the QLD depends on the efficiency of the cyclotron instability. For this purpose it is essential to consider the growth rate of the

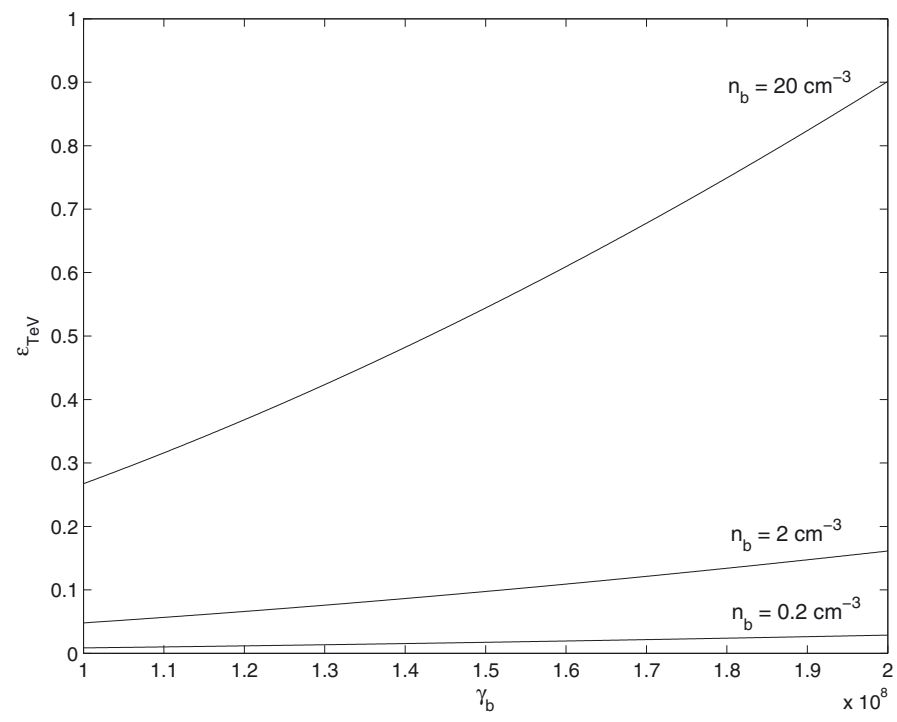

Fig. 4. Behaviour of $\epsilon_{\mathrm{TeV}}$ versus $\gamma_{\mathrm{b}}$. The set of parameters is $M_{9}=1$ and $B=10^{4} \mathrm{G}, \gamma_{\mathrm{p}}=2$ and $n_{\mathrm{b}}=\{0.2 ; 2 ; 20\} \mathrm{cm}^{-3}$.

instability. Kazbegi et al. (1992) showed that the increment, $\Gamma$, of the modes is given by

$\Gamma=\pi \frac{\omega_{\mathrm{b}}^{2}}{\omega \gamma_{\mathrm{p}}} \quad$ if $\quad \frac{1}{2} \frac{u_{x}^{2}}{c^{2}} \ll \delta$

and

$\Gamma=\pi \frac{\omega_{\mathrm{b}}^{2}}{2 \omega \gamma_{\mathrm{p}}} \frac{u_{x}^{2}}{\delta \cdot c^{2}} \quad$ if $\quad \frac{1}{2} \frac{u_{x}^{2}}{c^{2}} \gg \delta$,

where $\omega_{\mathrm{b}} \equiv \sqrt{4 \pi n_{\mathrm{b}} e^{2} / m}$ is the plasma frequency of resonant (beam) electrons. For the parameters $n_{\mathrm{b}}=20 \mathrm{~cm}^{-3}, \gamma_{\mathrm{p}}=2$ (see Fig. 1), by assuming $u_{x} \sim c$ and $\rho \sim R_{\mathrm{g}}$, one can show that $u_{x}^{2} /\left(2 c^{2}\right) \ll 1$, and the growth rate is defined by Eq. (15). Therefore, the corresponding cyclotron timescale, $t_{\mathrm{cyc}}=1 / \Gamma$, is of the order of $10^{-3} \mathrm{~s}$, which is less by many orders of magnitude than the kinematic timescale, $t_{\text {kin }} \sim R_{\mathrm{g}} / c \sim 10^{4} \mathrm{~s}$. The same relation between the timescales is valid for the rest of the considered cases; therefore, the studied mechanism is extremely efficient.

The interest in the QLD is twofold: on the one hand, the quasi-linear diffusion is an efficient mechanism that drives the synchrotron process despite very short synchrotron cooling timescales. On the other hand, as we have seen, the QLD provides the possibility of generating connected VHE $\gamma$-rays and radio emission on mpc scales. In particular, Bloom (2008) has found a high correlation of $\gamma$-ray luminosity and radio luminosity. The work of Giroletti et. al (2010) is particularly interesting, because they show that radio emission at $8.4 \mathrm{GHz}$ is connected to the VHE radiation $(>100 \mathrm{MeV})$. As we show in Fig. 5, the radio emission of the order of $8.4 \mathrm{GHz}$ is associated with $\gamma$-rays in the $\mathrm{GeV}$ band, therefore, the study of the QLD is very promising.

\section{Summary}

The main aspects of the present work can be summarized as follows:

1. We studied the role of the quasi-linear diffusion with magnetospheric plasma particles in AGNs. For this purpose we considered the kinetic equation that describes the balance between the synchrotron reaction force and the diffusion. 
Z. Osmanov: The generation of connected high and very high energy $\gamma$-rays and radio emission in active galactic nuclei

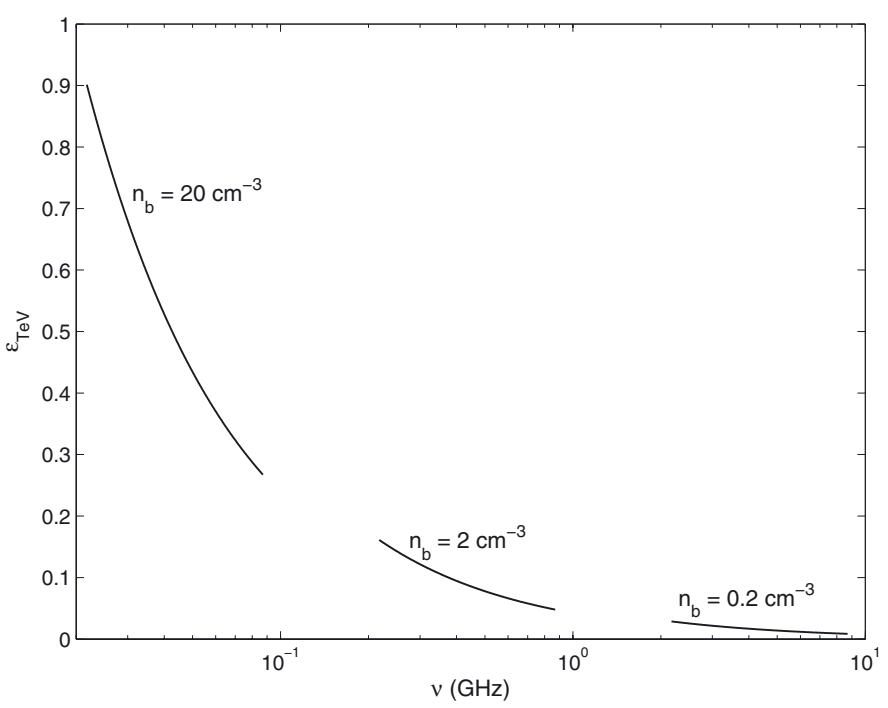

Fig. 5. Behaviour of $\epsilon_{\mathrm{TeV}}$ versus $v$. The set of parameters is $M_{9}=1$ and $B=10^{4} \mathrm{G}, \gamma_{\mathrm{p}}=2$ and $n_{\mathrm{b}}=\{0.2 ; 2 ; 20\} \mathrm{cm}^{-3}$. The curves are truncated for the same reason as in Fig. 3 .

2. We examined our model for different values of physical parameters. The QLD was studied versus the Lorentz factors of plasma and beam components respectively and versus the density of the beam component. We found that the higher the beam Lorentz factor, the higher the synchrotron emission energy and moreover, that the higher the plasma Lorentz factor, the lower the radio frequency. We also showed that lower beam densities lead to lower energies in the $\gamma$-ray synchrotron, and to higher energies in the radio domain.

3. We also found that under favourable conditions the cyclotron instability generates radio emission from $\sim 22 \mathrm{MHz}$ to $\sim 9 \mathrm{GHz}$ and the QLD provides synchrotron energies in the interval 900 down to $9 \mathrm{GeV}$. These results show the existence of connected radio and $\gamma$-ray emissions on mpc scales.

The QLD is a mechanism able to explain a connection between radio emission and VHE $\gamma$-rays. On the other hand, a complete study requires an investigation of the spectral pattern provided by the mentioned mechanism. According to the standard theory of the synchrotron emission (Bekefi \& Barrett 1977; Ginzburg 1981), it is assumed that along the line of sight the magnetic field is chaotic; correspondingly, the pitch angles vary in a broad interval (from 0 to $\pi$ ) and the particles have uniformly distributed pitch angles. Contrary to this scenario, in the framework of the present model the particles are nontrivially distributed (see Eq. (10)), therefore, the spectral picture will differ from those of Bekefi \& Barrett (1977), Ginzburg (1981). Another problem that has to be addressed is the variability of the corresponding VHE radiation. As the investigation shows, for the QLD to be efficient, two major requirements have to be fulfilled: the particles must be highly relativistic and the timescale of the cyclotron instability should be short enough. Therefore, the study of the variability implies the corresponding study of the acceleration timescale and the aforementioned instability timescale. We will investigate this problem in future studies.

Acknowledgements. The research was supported by the Georgian National Science Foundation grant GNSF/ST07/4-193. I also thank an anonymous referee for helpful suggestions.

\section{References}

Abert, J., Aliu, E., Anderhub, H., et al. 2008, ApJ, 674, 1037

Arav, N. K., Kirk, T., \& de Kool, M. 2002, ApJ, 566, 699

Bekefi, G., \& Barrett, A. H. 1977, Electromagnetic vibrations, waves and radiation (Cambridge, Massachusetts and London, England: The MIT Press)

Blandford, R. D., Netzer, H., \& Woltjer, L. 1990, Active Galactic Nuclei (Springer-Verlag)

Bloom, S. D. 2008, AJ, 136, 1533

Catanese, M., \& Weekes, T. C. 1999, PASP, 111, 1193

Chkheidze, N., \& Machabeli, G. 2007, A\&A, 471, 599

Chkheidze, N., Osmanov, Z., \& Machabeli, G. 2011, ApJ, 730, 62

Gangadhara, R. T. 1996, A\&A, 314, 853

Ginzburg, V. L. 1981, Teor. Fizika Astrofiz. (Nauka M.)

Giroletti, M., Reimer, A., Fuhrmann, L., et al. 2010 [arXiv: 1001.5123]

Kazbegi, A. Z., Machabeli, G. Z., \& Melikidze, G. I. 1991, MNRAS, 253, 377

Kovalev, Y. Y., Aller, H. D., Aller, M. F., et al. 2009, ApJ, 696, L17

Landau, L. D., \& Lifshitz, E. M. 1971, Classical Theory of Fields (London: Pergamon)

Levinson, A. 2000, Phys. Rev. L, 85, 912

Lominadze, J. G., Machabeli, G. Z., \& Mikhailovsky, A. B. 1979, J. Phys. Colloq., 40, 713

Machabeli, G., \& Osmanov, Z. 2009, ApJ, 700, L114

Machabeli, G., \& Osmanov, Z. 2010, ApJ, 709, 547

Machabeli, G. Z., \& Usov, V. V. 1979, AZhh Pis'ma, 5, 238

Malov, I. F., \& Machabeli, G. Z. 2001, ApJ, 554, 587

Osmanov, Z. 2010, ApJ, 721, 318

Osmanov, Z., \& Machabeli, G. 2010, A\&A, 516, 12

Osmanov, Z., Rogava, A. D., \& Bodo, G. 2007, A\&A, 470, 395

Rieger, F. M., \& Aharonian, F. A. 2008, A\&A, 479, L5

Rybicki, G. B., \& Lightman, A. P. 1979, Radiative Processes in Astrophysics (New York: Wiley)

Thomas, R. M. C., \& Gangadhara, R. T. 2005, A\&A, 437, 537

Thorne, K. S., Price, R. H., \& Macdonald, D. A. 1988, Black Holes: The Membrane Paradigm (New Haven: Yale University Press)

Xu, D., Komossa, S., Zhou, H., Tinggui, W., \& Jianyan, W. 2007, ApJ, 670, 60 